To be published in Optics Letters:

Title: $\quad$ Second harmonic illumination to enhance multispectral digital lensless holographic micros Authors: $\quad$ Omel Mendoza-Yero,Miguel Carbonell-Leal,Jesus Lancis,Jorge Garcia-Sucerquia Accepted: 03 February 16

Posted $\quad 04$ February 16

Doc. ID: 255038 


\title{
Second harmonic illumination to enhance multispectral digital lensless holographic microscopy
}

\author{
Omel Mendoza-Yero, ${ }^{1,}{ }^{*}$ Miguel Carbonell-Leal, ${ }^{1}$ Jesús Lancis, ${ }^{1}$ \\ AND JORGE GARCIA-SUCERQUIA ${ }^{2}$ \\ ${ }^{1}$ GROC•UJI, Institute of New Imaging Technologies, Universitat Jaume I, 12071-Castelló, Spain \\ ${ }^{2}$ Universidad Nacional de Colombia-Sede Medellin, School of Physics, A. A: 3840-Medellin, 050034, Colombia \\ *Corresponding author: omendoza@uji.es
}

Received XX Month XXXX; revisedXX Month, XXXX; accepted XX MonthXXXX; posted XX Month XXXX (Doc. ID XXXXX); published XX Month XXXX

\begin{abstract}
Multispectral digital lensless holographic microscopy (MDLHM) operating with second harmonic illumination is shown. Additionally to the improvement of the spatial resolution of the previously reported MDLHM operating with near-infrared illumination, this second harmonic MDLHM shows promising as a tool to study the behavior of biological samples under a broad spectral illumination. This illumination is generated by focusing a highly spatially coherent ultrashort pulsed radiation into a BBO nonlinear crystal. The second harmonic MDLHM allows achieving multispectral images of biological samples with enhanced micrometer spatial resolution. The illumination wavelength of the second harmonic MDLHM can be tuned by displacing a focusing optics with respect to a pinhole; spatially resolved information at different wavelengths of the sample can be then retrieved. (C) 2016 Optical Society of America
\end{abstract}

OCIS codes: (090.1995) Digital holography; (110.0180) Microscopy; (110.4234) Multispectral and hyperspectral imaging; (190.7110) Ultrafast nonlinear optics.

http://dx.doi.org/10.1364/OL.99.099999

Digital lensless holographic microscopy (DLHM) requires a point source and a digital camera to provide images of the micro world [1,2] This simplicity of hardware has promoted its use in different scenarios, among which can be counted the utilization of multiple type of point sources. The most versatile version of DLHM is perhaps that based on multiple illumination wavelengths to acquire spectral resolved images of microscopic samples. Full color and wavelength dependent images of biological samples have been presented with the use of what has been coined as multispectral digital lensless holographic microscopy (MDLHM) [3-6].

This microscopy technique has been implemented utilizing a multiwavelength illumination, provided by spatially coherent or incoherent broadband spectral light sources, or by a set of quasi-monochromatic lasers, or alternatively by a single spectrally tunable laser, to enable the synthesizing of multispectral images. In this context, the information on the spatially distributed frequency emissions extracted from multispectral images eases to discriminate among several cell's components like proteins or chromosomes, or to approach their physical relations, functions, and progression over time [7, 8]. Additionally, a suitable set of images corresponding to emission data of a biological sample for different spectral lines named as lambda stack or spectral image cube [9] can be utilized to separate spectral contributions from multiple dyes that overlap not only spectrally but also spatially.

Closely related, multispectral diffractive imaging has been investigated as a way of low temporal coherence, high spatial coherence optical field produced by high-harmonic generation sources in X-ray imaging [10-12]. Further, the use of white-light sources has been demonstrated in diffraction phase microscopy to promote enhanced spatial phase sensitivity with improved signal-to-noise ratio [13].

Despite the great advance that has been reached with the use of tunable broadband lasers in MDLHM, the spatial resolution of the multispectral images reported with this technique is still to be improved. In MDLHM the spatial resolution $\Delta r$ of the reconstructed images is given by

$$
\Delta r=\frac{\lambda}{2 N A}
$$

with $\lambda$ the illuminating wavelength of a fully spatial coherent source and $N A$ the numerical aperture of the microscope. The latter is determined by the width $W$ of the recording digital camera, and the distance of its center from the point source $L$, such that $N A \cong W /\left\{2\left[(W / 2)^{2}+L^{2}\right]^{1 / 2}\right\}$. As the illuminating source is partially spatially coherent, the spatial resolution is no longer controlled exclusively by Eq. (1). In addition, the effective size of the point source plays a key role on determining the size of the objects that can be differentiated with MDLHM; the smaller the size of the point source the smaller $\Delta r$ that can be achieved [14].

By using as illuminating source a Ti: Sa femtosecond laser that emits pulses of about $12 \mathrm{fs}$ intensity full width at half maximum (FWHM), operating at $\lambda_{0}=800 \mathrm{~nm}$ central wavelength, with $85 \mathrm{~nm}$ intensity FWHM spectral bandwidth, a MDLHM has been proposed [5]. In that 
set-up, the point source was generated by focusing down the light from laser over a pinhole by means of a kinoform diffractive lens (DL). In this configuration, by changing the distance between the DL and the pinhole plane, one can select very narrow wavelength bands within the whole spectrum of the incident light. Although, this diffractive-based microscopy set-up enables the production of a suited set of lambda stack images, it is clear that their spatial resolution is limited by the long wavelengths content of the used illumination source. This result points out the need for broadband illuminating sources operating at smaller wavelengths that can be utilized in MDLHM.

In this letter, we present a MDLHM set-up that uses a second harmonic (SH) generated light source to improve the spatial resolution of the technique. The use of SH field as a contrast agent for optical imaging of biological samples through off-axis digital holography was first proposed in [15]. SH generation nanoprobes can be used for in vivo imaging and circumvent many of the limitations of classical fluorescence probes [16]. Also, SH has been used for label-free imaging of endogenous features of biological samples like muscle tissue [1719]. In our proposal, spatially coherent second harmonic illumination, generated by focusing ultrashort near-infrared pulses within a nonlinear crystal, is utilized to obtain multispectral images of a paramecium with enhanced spatial resolution, without the use of lenses as imaging system.

In Fig. 1, a schematic diagram of the optical set-up utilized for improving the spatial resolution in MDLHM is shown.

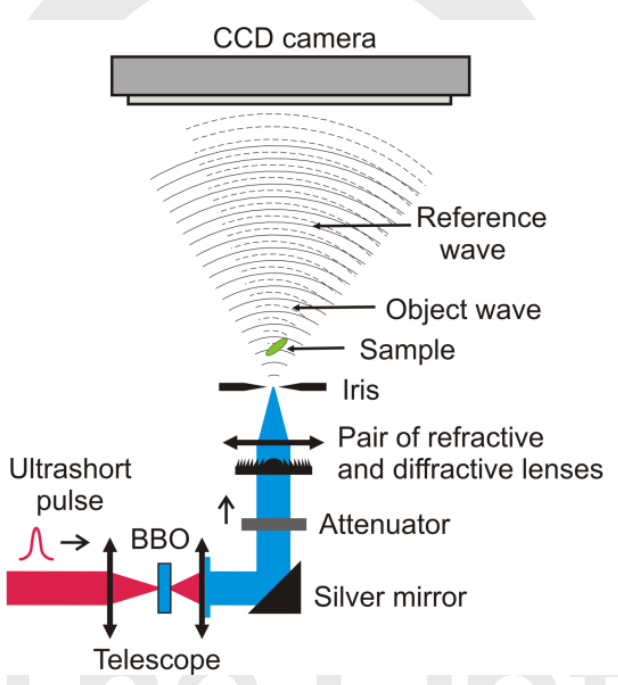

Fig. 1. (Color online) Schematic representation of the second harmonic illumination MDLHM setup for getting multispectral images

The light source is a Ti: Sa femtosecond laser similar to the one used in [5], whose main characteristics were above-mentioned. The SH beam is generated by focusing the light from the pulsed laser, with the help of an achromatic refractive lens of $50 \mathrm{~mm}$ focal length, into an uncoated type-I $\beta-\mathrm{BaB}_{2} \mathrm{O}_{4} \quad(\mathrm{BBO})$ crystal with dimensions $10 \mathrm{~mm} \times 10 \mathrm{~mm} \times 0.02 \mathrm{~mm}$ ). After the BBO crystal, the light is both recollimated by using another achromatic refractive lens of $200 \mathrm{~mm}$ focal length, and conveniently filtered to suppress unconverted infrared wavelengths. The thickness of the crystal ( $20 \mu \mathrm{m}$ ) guaranties full conversion of wavelengths due to phase-matching condition. The $\mathrm{SH}$ beam is expanded by a telescope to fill the whole area of the kinoform DL, which is about $350 \mathrm{~mm}^{2}$. Then, the SH beam is focused down onto a pinhole with diameter $d=1 \mu \mathrm{m}$ with the help of two lenses. The first lens is a kinoform DL (Novosibirsk, Rusia), optimized to focus a radiation of $565.1 \mathrm{~nm}$ in a plane located $150 \mathrm{~mm}$ away from it, whereas the second is a refractive lens with focal length of $80 \mathrm{~mm}$. Here, it should be mentioned that the combination of diffractive and refractive lens allows the reduction of the size of the focused spot, which improves the throughput of the pinhole, in comparison with the use of a single DL. This improvement of the light gathered after the pinhole is particular important in the proposed setup because the available amount of SH signal is mainly determined by the conversion efficiency of the nonlinear crystal, which in our conditions was of the order of $64 \%$.

The sample, placed a fixed distance $z_{0}=2 \mathrm{~mm}$ from the pinhole plane, is illuminated with a SH spherical wave, as shown in Fig. 1. The light scattered by the sample is partially coherently superimposed on the surface of the digital camera with the portions of the spherical wave that travel without perturbation. The recorded intensity, named in-line hologram, is recorded by a CCD Basler A120f digital camera located at a distance $L=10 \mathrm{~mm}$ from the pinhole plane; this configuration provides a theoretical spatial resolution of $\Delta r \cong 628 \mathrm{~nm}$ for central wavelength of the SH illumination. The intensity measured without sample is pixel-wise subtracted from the in-line hologram. To reconstruct the wavefront scattered by the sample, the resulted intensity is numerically processed by means of a specific software based on complex wave field propagation through the FresnelKirchhoff diffraction integral. The use of spherical illumination ensures that the reconstructed images do not suffer from any nuisance due to the presence of the twin images [20]. The utilized hologram reconstruction process based on a circular convolution [21], also guaranties the size of the image coordinates at the reconstruction plane to be independently of the wavelength. This feature allows the corrected sizing of the different reconstructions for the set of illuminating wavelengths in such a way all the reconstructed images have the same scale factor. Further details of the complete image reconstruction process can be found elsewhere [21]. Here, it should be also noted that other techniques have been introduced for controlling the size of the reconstructed images in multi-wavelength digital holography $[22,23]$. Despite of its correctness, we have chosen the circular convolution because it keeps the number of pixels constant maintaining also constant the computation time.

In this work, a paramecium (29-6914 Carolina) was used as a sample. Its length is approximately $220 \mu \mathrm{m}$. In the middle-left part of Fig. 2, an image of the reconstructed hologram for the spectral line $400 \mathrm{~nm}$ is shown. 


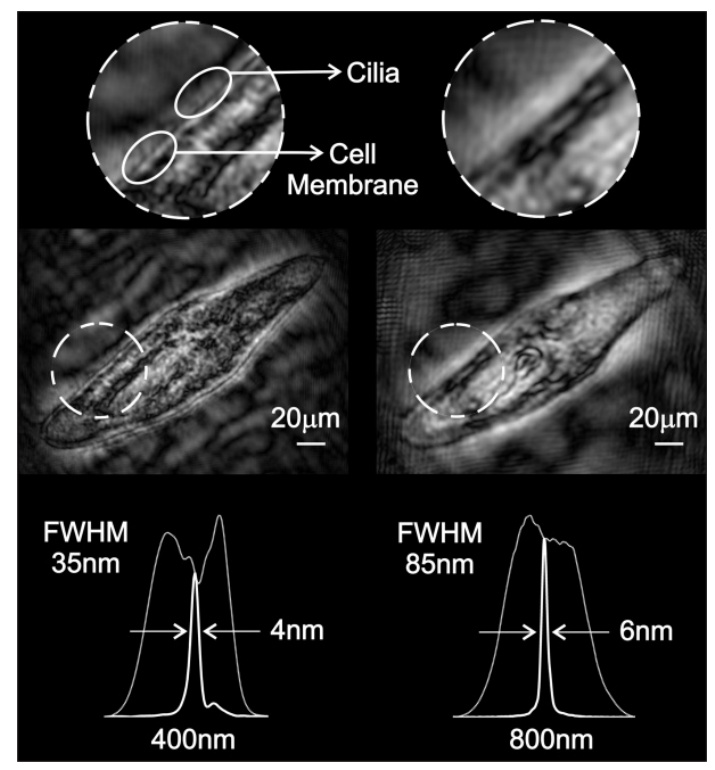

Fig. 2. Reconstructed hologram of a paramecium for the spectral lines centered at $400 \mathrm{~nm}$ (middle-left part) , and $800 \mathrm{~nm}$ (middle -right part). Details (zoomed in) of the paramecium structure (top part). Spectral profiles of the second harmonic and initial ultrashort light, together with their corresponding selected spectral lines (bottom part).

Some structures of the paramecium with dimensions in the order of two micrometers e.g., cell membrane or cilia, are highlighted in the topleft part of Fig. 2 as an inset. This inset is obtained by 1.6x magnifying the encircle region of the image of the paramecium. In the bottom-left part of Fig. 2, the profile of the second harmonic spectrum together with the selected spectral line of $4 \mathrm{~nm}$ intensity FWHM width are also shown. In the middle -right part of Fig. 2, the reconstructed image of same sample, but imaged with a spectral line $800 \mathrm{~nm}$, is shown. To do that, the nonlinear crystal was removed from the set-up, and the position of the pair of diffractive-refractive lenses was slightly modified to select the spectral line in the vicinity of $800 \mathrm{~nm}$. The magnified encircle region that appears in the top-right part of Fig. 2 also corresponds to the same portion of the image. Note that, now the structures corresponding to the cell membrane or cilia are almost indiscernible from this encircle region. By using the matlab function called "measerr", one can determine that the mean square difference between the images obtained in both cases yields $3.5 \%$. After a visual comparison of images reconstructed for the central wavelengths $400 \mathrm{~nm}$ and $800 \mathrm{~nm}$, respectively, one can conclude that second harmonic illumination allows achieving an image with clearly enhanced spectral resolution.

In order to show the ability of the proposed MDLHM set-up to obtain high quality images of a biological sample at different wavelength bands, we carry out a spectral tuning of the second harmonic light. To do that we slightly change the axial distance between the pinhole plane and the plane of the focusing optics by using a micrometer screw gauge. The focusing optics is composed of a pair of diffractive-refractive lenses that can be jointly moved along the axial direction of the microscope. The combined utilization of the pinhole (iris) and the pair of diffractive and refractive lenses can be regarded as a spectrometer. That is, as the focal length of the focusing optics varies inversely with the wavelength of the incident light producing strong chromatic aberrations, one can select narrow spectral lines by using a simple pinhole (iris) that operates as a spatial filter. Hence, by placing the pinhole at that particular position, spherical waves with specific narrow wavelength contents are produced. To record the spectral lines corresponding to each displacement of the join lenses, the CCD camera was temporally substituted by a commercial spectrometer. Following this procedure, seven spectral lines, stepped $5 \mathrm{~nm}$ from $385 \mathrm{~nm}$ to 415 $\mathrm{nm}$ were obtained. To measure the intensity of corresponding spherical waves without sample, the camera was restored into its original place and the focusing optics are displaced to the same axial positions as before. At these positions, after introduced the sample into the MDLHM set-up, the corresponding in-line holograms are recorded. In Fig. 3, a multispectral image of the same paramecium sample is shown.

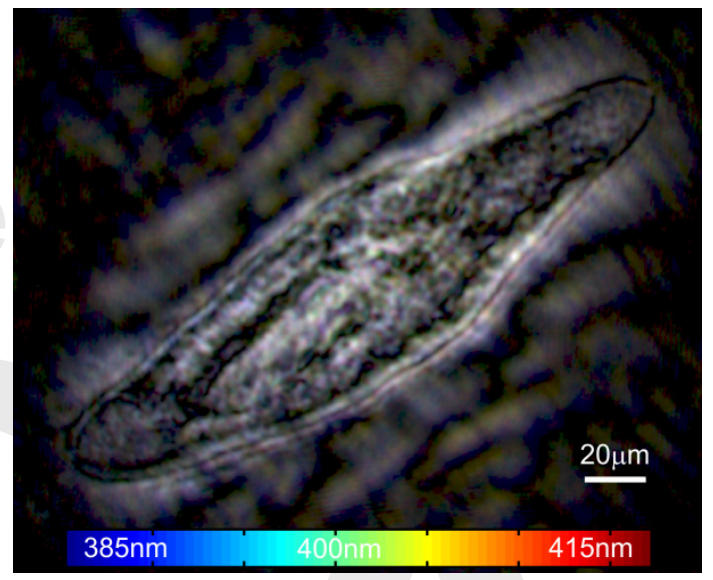

Fig. 3. (Color online) Pseudo colored RGB image of a paramecium obtained from the merging of images corresponding to the spectral lines $415 \mathrm{~nm}, 400 \mathrm{~nm}$, and $385 \mathrm{~nm}$, respectively.

To generate this image, we merged three images corresponding to the spectral lines 385, 400, and $415 \mathrm{~nm}$, respectively. In the resulted pseudo-colored RGB image the red channel was formed with the reconstructed image for $415 \mathrm{~nm}$, the green channel with the corresponding one for $400 \mathrm{~nm}$, whereas the remaining blue channel with the image obtained for $385 \mathrm{~nm}$. The spectral lines from the second harmonic spectrum that were used to illuminate the sample are included in the color-bar located at bottom part of Fig. 3.

From Fig. 3, one can see that most regions of the paramecium show an even response to the different wavelength content. However, some other regions of are wavelength-dependent, with preference for the $385 \mathrm{~nm}$. The predominant white color of the image might be determined by the relative small bandwidth of the second harmonic light, which is approximately $35 \mathrm{~nm}$ intensity FWHM. The combination of other three images, corresponding to different spectral lines, can originate another multispectral image, containing new information on the absorption/emission distribution of frequencies. This fact, together with the possibility of tuning a spectral line along the whole spectrum of the illuminating source, turn the proposed MDLHM set-up into a friendly optical system for spectral analysis of biological samples.

Our results experimentally show that second harmonic generation from a spatially coherent broadband light can be very suited for MDLHM applications. In this case, image reconstruction can be beneficiated not only from the high spatial coherent of the initial light source, but also from the frequency-double content of the second harmonic spectrum, which positively contribute to obtain image with enhanced spatial resolution, as seen in Fig. 2. 
Funding. Ministerio de Economía y Competitividad (MINECO) (grant FIS2013-40666-P), Generalitat Valenciana (PROMETEO 2012-021, and ISIC 2012/013), Universitat Jaume I (P1-1B2012-55); Youth research program, Colciencias-Universidad Nacional de Colombia (Hermes 28751).

Acknowledgment. The authors are also very grateful to the SCIC of the Universitat Jaume I for the use of the femtosecond laser.

\section{References}

1. W. Xu, M.H. Jericho, I. A. Meinertzhagen, and H. J. Kreuzer, Proc. Nat. Acad. Sci. 98, 11301 (2001).

2. J. Garcia-Sucerquia, W. Xu, S. K. Jericho, P. Klages, M. H. Jericho, and H. J. Kreuzer, Appl. Opt. 45, 836 (2006).

3. J. P. Ryle, S. McDonnell, and J. T. Sheridan, J. Biomed. Opt. 16, 126004 (2011).

4. J. P. Ryle, K. M. Molonyd, S. McDonnelle, T. J. Naughtond, and J. T. Sheridan, Proc. of SPIE. 7442, 744206 (2009).

5. O. Mendoza-Yero, E. Tajahuerce, J, Lancis, and J. Garcia-Sucerquia, Opt. Lett. 38, 2107 (2013).

6. J. Garcia-Sucerquia, Opt. Lett. 37, 1724 (2012).

7. M. Brydegaard, A. Merdasa, H. Jayaweera, J. Alebring, and S. Svanberg, Rev. Sci. Instrum. 82, 23106 (2011).

8. M. E. Dickinson, G. Bearman, S. Tille, R. Lansford, and S. E. Fraser, Bioimaging 31, 1272 (2001).

9. R. Lansford, G. Bearman, and S. E. Fraser, J. Biomed. Opt. 6, 311 (2001).

10. B. Chen, R. A. Dilanian, S. Teichmann, B. Abbey, A. G. Peele, G. J. Williams, P. Hannaford, L. Van Dao, H. M. Quiney, and K. A. Nugent, Phys. Rev. A 79, 023809 (2009).

11. B. Abbey, L. W. Whitehead, H. M. Quiney, D. J. Vine, G. A. Cadenazzi, C. A. Henderson, K. A. Nugent E. Balaur, C. T. Putkunz, A. G. Peele, G. J. Williams, and I. McNulty, Nature Photon 5, 420 (2011).

12. S. Witte, V. T. Tenner, D. W. Noom, and K. S. Eikema, Light Sci Appl 3, e163 (2014).

13. B. Bhaduri, H. Pham, M. Mir, and G. Popescu, Opt. Lett. 37, 1094 (2012).

14. J. Garcia-Sucerquia, App. Opt. 52, A232 (2013).

15. Y. Pu, M. Centurion, and D. Psaltis, Appl. Opt. 47, A103 (2008).

16. P. Pantazis, J. Maloney, and D. Wu, Proc. Nat. Acad. Sci. 107, 14535 (2010).

17. O. Masihzadeh, P. Schlup, and R. A. Bartels, Opt. Express. 18, 9840 (2010).

18. E. Shaffer, C. Moratal, P. Magistretti, P. Marquet, and C. Depeursinge, Opt. Lett. 35, 4102 (2010).

19. C. Macias-Romero, M. E. P. Didier, P. Jourdain, P. Marquet, P. Magistretti, O. B. Tarun, V. Zubkovs, A. Radenovic, and S. Roke, Opt. Express 22, 31102 (2014).

20. J. Garcia-Sucerquia, D. C. Alvarez-Palacio, M. H. Jericho, H. J. Kreuzer, Opt. Lett. 31, 2845 (2006).

21. M. H. Jericho and H. J. Kreuzer, in Coherent Light Microscopy, P. Ferraro, A. Wax, and Z. Zalevvsky, eds. (2011), pp. 3-30.

22. D. Alfieri, G. Coppola, S. De Nicola, P. Ferraro, A. Finizio, G. Pierattini, and B. Javidi, Opt. Comm. 260, 113-116 (2006).

23. S. De Nicola, A. Finizio, G. Pierattini, D. Alfieri, S. Grilli, L. Sansone, and P. Ferraro, Opt. Lett. 30, 2706-2708 (2005). 


\section{Complete references}

1. W. Xu, M. H. Jericho, I. A. Meinertzhagen, and H. J. Kreuzer, "Digital in-line holography for biological applications," Proc. Nat. Acad. Sci. 98, 11301-11305 (2001).

2. J. Garcia-Sucerquia, W. Xu, S. K. Jericho, P. Klages, M. H. Jericho, and H. J. Kreuzer, "Digital in-line holographic microscopy," Appl. Opt. 45, 836-850 (2006).

3. J. P. Ryle, S. McDonnell, and J. T. Sheridan, "Lensless multispectral digital in-line holographic microscope," J. Biomed. Opt. 16, 126004 (2011).

4. J. P. Ryle, K. M. Molonyd, S. McDonnelle, T. J. Naughtond, and J. T. Sheridan, "Multispectral lensless digital holographic microscope: imaging MCF-7 and MDA-MB-231 cancer cell cultures," Proc. of SPIE. 7442, 744206 (2009).

5. O. Mendoza-Yero, E. Tajahuerce, J, Lancis, and J. Garcia-Sucerquia, "Diffractive digital lensless holographic microscopy with fine spectral tuning," Opt. Lett. 38, 2107-2109 (2013).

6. J. Garcia-Sucerquia, "Color lensless digital holographic microscopy with micrometer resolution," Opt. Lett. 37, 1724-1726 (2012).

7. M. Brydegaard, A. Merdasa, H. Jayaweera, J. Alebring, and S. Svanberg, "Versatile multispectral microscope based on light emitting diodes," Rev. Sci. Instrum. 82, 23106 (2011).

8. M. E. Dickinson, G. Bearman, S. Tille, R. Lansford, and S. E. Fraser, "Multi-spectral Imaging and Linear Unmixing Add a Whole New Dimension to Laser Scanning Fluorescence Microscopy," Bioimaging 31, 1272-1278 (2001).

9. R. Lansford, G. Bearman, and S. E. Fraser, "Resolution of multiple green fluorescent protein color variants and dyes using two-photon microscopy," J. Biomed. Opt. 6, 311-318 (2001).

10. B. Chen, R. A. Dilanian, S. Teichmann, B. Abbey, A. G. Peele, G. J. Williams, P. Hannaford, L. Van Dao, H. M. Quiney, and K. A. Nugent, "Multiple wavelength diffractive imaging," Phys. Rev. A 79, 023809 (2009)

11. B. Abbey, L. W. Whitehead, H. M. Quiney, D. J. Vine, G. A. Cadenazzi, C. A. Henderson, K. A. Nugent E. Balaur, C. T. Putkunz, A. G. Peele, G. J. Williams, and I. McNulty, "Lensless imaging using broadband X-ray sources," Nature Photon 5, 420-424 (2011).

12. S. Witte, V. T. Tenner, D. W. Noom, and K. S. Eikema, "Lensless diffractive imaging with ultra-broadband table-top sources: from infrared to extreme-ultraviolet wavelengths," Light Sci Appl 3, e163-8 (2014).

13. B. Bhaduri, H. Pham, M. Mir, and G. Popescu, "Diffraction phase microscopy with white light," Opt. Lett. 37, 1094-1096 (2012).

14. J. Garcia-Sucerquia, "Noise reduction in digital lensless holographic microscopy by tailoring the light from a light-emitting diode," App. Opt. 52, A232-A239 (2013).

15. Y. Pu, M. Centurion, and D. Psaltis, "Harmonic holography: a new holographic principle," Appl. Opt. 47, A103 (2008).

16. P. Pantazis, J. Maloney, and D. Wu, "Second harmonic generating (SHG) nanoprobes for in vivo imaging," Proc. Nat. Acad. Sci. 107, 14535-14540 (2010).

17. O. Masihzadeh, P. Schlup, and R. A. Bartels, "Labelfree second harmonic generation holographic microscopy of biological specimens," Opt. Express. 18, 9840-9851 (2010).

18. E. Shaffer, C. Moratal, P. Magistretti, P. Marquet, and C. Depeursinge, "Label-free second-harmonic phase imaging of biological specimen by digital holographic microscopy," Opt. Lett. 35, 4102-4104 (2010).

19. C. Macias-Romero, M. E. P. Didier, P. Jourdain, P. Marquet, P. Magistretti, O. B. Tarun, V. Zubkovs, A. Radenovic, and S. Roke "High throughput second harmonic imaging for label-free biological applications," Opt. Express 22, 31102-31111 (2014).

20. J. Garcia-Sucerquia, D. C. Alvarez-Palacio, M. H. Jericho, H. J. Kreuzer, "Comment on "Reconstruction algorithm for high-numerical aperture hologram with diffraction limited resolution", Opt. Lett. 31, 2845-2847 (2006).

21. M. H. Jericho and H. J. Kreuzer, "Point Source Digital In-line Holographic Microscopy," in Coherent Light Microscopy, P. Ferraro, A. Wax, and Z. Zalevvsky, eds. (2011), pp. 3-30.

22. D. Alfieri, G. Coppola, S. De Nicola, P. Ferraro, A. Finizio, G. Pierattini, and B. Javidi, "Method for superposing reconstructed images from digital holograms of the same object recorded at different distance and wavelength," Opt. Comm. 260, 113-116 (2006).

23. S. De Nicola, A. Finizio, G. Pierattini, D. Alfieri, S. Grilli, L. Sansone, and P. Ferraro, "Recovering correct phase information in multiwavelength digital holographic microscopy by compensation for chromatic aberrations," Opt. Lett. 30, 2706-2708 (2005). 